\title{
Differential surface competition and biofilm invasion strategies of Pseudomonas aeruginosa PA14 and PA01
}

${ }^{\dagger}$ These authors contributed equally to this work.

${ }^{1}$ Department of Microbiology and Immunology, Geisel School of Medicine at Dartmouth, 13 Hanover, $\mathrm{NH}$

$14{ }^{2}$ Department of Biology, Dartmouth College, Hanover, NH

*To whom correspondence should be addressed

19 Carey D. Nadell

20 Rm 326 Class of 1976 Life Science Center

2178 College St.

22 Dartmouth, Department of Biological Sciences

23 Hanover, NH 03755

24 Phone: (603) 6461525

25 email: carey.d.nadell@ dartmouth.edu

George A O'Toole

Rm 202 Remsen Building

66 College Street

30 Geisel School of Medicine at Dartmouth

31 Hanover, NH 03755

32 Phone: (603) 650-1248 (office)

33 email:georgeo@Dartmouth.edu

Running title: PAO1-PA14 competition

Key words: Pseudomonas aeruginosa, biofilm, exopolysaccharide, competition, starvation 


\section{$\underline{\text { Abstract }}$}

$40 \quad$ Pseudomonas aeruginosa strains PA14 and PAO1 are among the two best characterized model

41 organisms used to study the mechanisms of biofilm formation, while also representing two

42 distinct lineages of $P$. aeruginosa. Our previous work showed that $P$. aeruginosa PA14 and

43 PAO1 use distinct strategies to initiate biofilm growth. Using differentially-labeled strains and

44 microfluidic devices, we show that PAO1 can outcompete PA14 in a head-to-head competition

45 during early colonization of a surface, can do so in constant and perturbed environments, that

46 this advantage is specific to biofilm growth and requires production of the Psl polysaccharide. In

47 contrast, the $P$. aeruginosa PA14 exhibits a competitive fitness advantage when invading a pre-

48 formed biofilm and is better able to tolerate starvation than PAO1 in the biofilm context. These

49 data support the model that while P. aeruginosa PAO1 and PA14 are both able to effectively

50 colonize surfaces, these strains use distinct strategies that are advantageous under different

51 environmental settings.

\section{Importance}

54 Recent studies indicate that $P$. aeruginosa PAO1 and PA14 use distinct strategies to initiate 55 biofilm formation, with PAO1 committing to the surface through a processive mode of 56 attachment, while PA14 uses a non-processive surface engagement strategy. We investigated

57 whether their respective colonization strategies impact their ability to effectively compete under 58 different biofilm-forming regimes. Our work shows that these different strategies do indeed 59 impact how these strains colonize the surface: PAO1 dominates during colonization of a naïve 60 surface, while PA14 is more effective in colonizing a pre-formed biofilm or withstanding 61 starvation conditions. These data suggest that even for very similar microbes there may be 62 distinct strategies to successfully colonize and persist on surfaces during the biofilm life cycle.

\section{Introduction}

65 Biofilms are surface-attached microbial communities that mediate long-term growth and 66 persistence on substrates as varied as vegetable produce and indwelling medical devices $(1,2)$.

67 Biofilms are typically polymicrobial in nature and exhibit an increased tolerance to antimicrobial 68 agents, predation and reactive oxygen species (3-5). The transition from planktonic to biofilm 69 modes of growth is complex and uses signaling systems initiated by surface engagement that 
70 have downstream consequences for biofilm formation (6-8). Our understanding of biofilm

71 formation comes in part from the study of Pseudomonas aeruginosa, a Gram-negative bacterium

72 that forms prolific biofilms in aquatic environments, soils and clinical settings (9-12). To form a

73 biofilm, motile bacteria such as $P$. aeruginosa use appendages including flagella and pili to

74 mediate early surface engagement $(13,14)$. Surface colonization and growth during biofilm

75 formation are also linked to extracellular polysaccharide (EPS) production, which provides cell-

76 to-surface and cell-cell adhesion (15). EPS is required for early attachment events, particularly in

77 the PAO1 lineage, as well as microcolony development; this matrix material also plays a key role

78 in providing the structural integrity required for mature biofilms to form and persist $(16,17)$.

79 Current evidence suggests that $P$. aeruginosa use two distinct signaling systems to

80 initiate formation of a biofilm $(18,19)$ : the second messenger c-di-GMP, which is regulated

81 through the Wsp system (20-22), and the second messenger c-AMP (cAMP), which is regulated

82 through the Pil-Chp/Vfr system $(23,24)$. For $P$. aeruginosa PAO1, upon surface attachment,

83 membrane-bound WspA is thought to behave as a sensor of surface contact, contributing to the

84 phosphorylation and activation of the diguanylate cyclase WspR $(22,25)$ and resulting in

85 increased c-di-GMP synthesis (26). In contrast, for P. aeruginosa PA14, signaling via the Pil-

86 Chp system is initiated through type IV pili (TFP) engaging a surface, which in turn transmits

87 this surface signal to the adenylate cyclase, CyaB. The activation of CyaB increases

88 concentrations of cAMP, resulting in production and secretion of cell surface-localized PilY1.

89 The PilY1 protein participates in the activation of diguanylate cyclase SadC by an unknown

90 mechanism to enhance c-di-GMP production (24, 27-29). Thus, both of these pathways

91 ultimately increase c-di-GMP level and enhance biofilm formation.

92 The two common laboratory strains of $P$. aeruginosa, abbreviated PAO1 and PA14,

93 represent two distinct lineages based on whole genome phylogenetic analysis (30). PAO1 and

94 PA14 preferentially use the WspR and Pil-Chip signaling pathways to enhance EPS production

95 and repress surface motility, respectively, during early events in biofilm formation (24, 31).

96 Previously, we have suggested that PAO1 uses the Wsp system to facilitate a processive regime

97 of attachment that results in an early commitment to a surface (19). In contrast, PA14 is non-

98 processive, such that cells are less committed after initial surface engagement while their

99 progeny are primed for subsequent reattachment through a cAMP-dependent signaling pathway

$100(19,32)$. 
The respective strategies by which PAO1 and PA14 are able to attach to a surface provide a useful model system by which we can test how initial colonization strategies influence competitiveness in the biofilm context when both strains are grown together, and thus compete

104 for space and nutrients. The different surface-attachment strategies of PAO1 and PA14 may also

105 provide insights in understanding how various bacterial genera, species and strains cooperate and/or compete to colonize surfaces in polymicrobial environments.

\section{$\underline{\text { Results }}$}

\section{PAO1 outcompetes PA14 in dual-strain biofilms}

112 To test if the colonization strategies used by $P$. aeruginosa PA14 and $P$. aeruginosa PAO1 113 (abbreviated as PA14 and PAO1, respectively) impart an advantage during the early stages of 114 biofilm formation (i.e., initial colonization of a clean surface), we used microfluidic devices in 115 which bacteria can be monitored as they attach and grow on glass substrata. To allow for 116 visualization of each strain by widefield or confocal fluorescence microscopy, constitutive 117 fluorescent protein expression constructs were introduced, with a single copy of GFP 118 (ex500/em513) and two copies of $m K O$ (ex548/em559) inserted at the att site of PAO1 and 119 PA14, respectively (33).

121 To assess competition during early biofilm formation, PAO1 and PA14 were inoculated into 122 microfluidic chambers in a 1:1 ratio and imaged over 7 hours of flow in a buffered minimal 123 medium containing $1.0 \mathrm{mM} \mathrm{K}_{2} \mathrm{HPO}_{4}, 0.6 \mathrm{mM} \mathrm{MgSO}_{4}$ and $0.4 \%$ arginine. We normalized the 124 number of cells for each strain to the number of cells at the start of the assay (0h) and measured 125 the fold change in cell number over time. PAO1 showed a significant advantage in biofilm 126 growth over PA14 as early as four hours after surface inoculation; after 7h of incubation, the 127 number of PAO1 cells was $~ 2.5$-fold greater than that of PA14 (Fig. 1A, B, C).

129 If the observed advantage of PAO1 during biofilm competition was due to enhanced growth rate 130 or antagonistic interactions with PA14 via diffusible secreted factors, one would expect a similar 131 competitive outcome in mixed liquid culture conditions. To test this possibility we grew 
132 planktonic PAO1 and PA14 in glass tubes containing sterile biofilm medium either in mono133 culture or 1:1 co-culture. PAO1 and PA14 grew equally well when cultured separately (Fig.

134 S1A), and neither strain outgrew the other in mixed planktonic cultures (Fig. S1B). Notably, the

135 CFU counts of the strains in a co-culture were lower by equal measures than their respective 136 CFU counts when grown alone; this indicates neutral competition in which the two strains 137 compete for limited resources in mixed liquid co-culture, but neither has an advantage over the 138 other. These results demonstrate that the advantage in biofilm competition for PAO1 is not due 139 to higher basal growth rate but rather to other root causes.

141 Biofilms often exhibit complex architectures that can vary significantly in mixed strain or mixed 142 species contexts $(34,35)$; in light of these observations we next assessed the spatial structure of 143 PAO1 and PA14 in biofilm co-culture. To monitor these dynamics, PAO1 and PA14 were grown 144 together in microfluidic chambers for $24 \mathrm{~h}$ and then imaged by confocal microscopy. Consistent 145 with the pattern observed in early biofilms inoculated with a 1:1 ratio of PAO1 and PA14 (Fig. 146 1A,1B), after 24h of growth PAO1 showed a significant increase in relative abundance compared 147 to PA14 (Fig. 1D,1G). Z-projections of confocal image stacks revealed that PAO1 grows across 148 the top of the PA14 cell clusters (Fig. 1D, bottom panel; Fig. 1E,1F), an observation consistent 149 with a previous report from Diggle and colleagues (36). The above data provide evidence that when grown in the biofilm context, PAO1 has an early 152 fitness advantage against PA14 and can ultimately dominate the population. Thus far, a 1:1 153 inoculation ratio of PAO1 to PA14 was used to assess dual strain population dynamics. This 154 condition does not account for the possibility of frequency-dependent competition, in which the 155 favored strain may depend on the initial ratio of the two. To test if the competitive advantage of 156 PAO1 was frequency-dependent, we varied the starting PAO1 frequency from 0.1 through 0.9 157 and measured the change in frequency of PAO1 versus PA14 after 24h. Regardless of the 158 starting frequency, PAO1 consistently increased in relative abundance (Fig. 1H, Fig. S2A,S2B). 159 We also tested whether starting density would affect this dynamic and found that irrespective of 160 the tested starting densities, PAO1 was able to increase in frequency in a dual-strain biofilm 161 (Fig. S2C). We conclude from these experiments that under flow in the conditions used here, 162 PAO1 outcompetes PA14 in a frequency- and density-independent fashion. 
PAO1 PA14

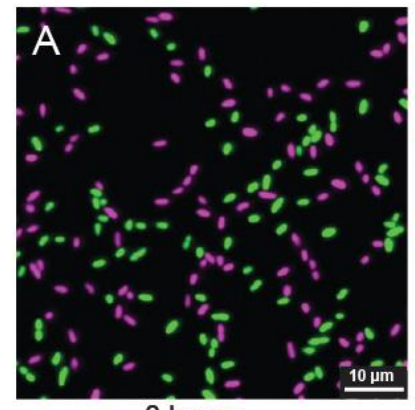

0 hours
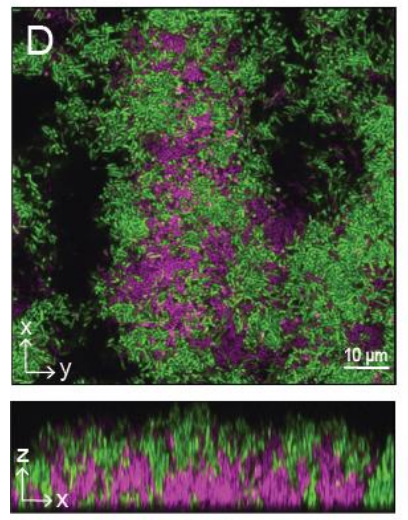

24 hours

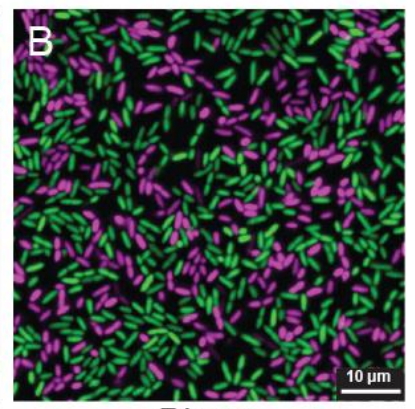

7 hours
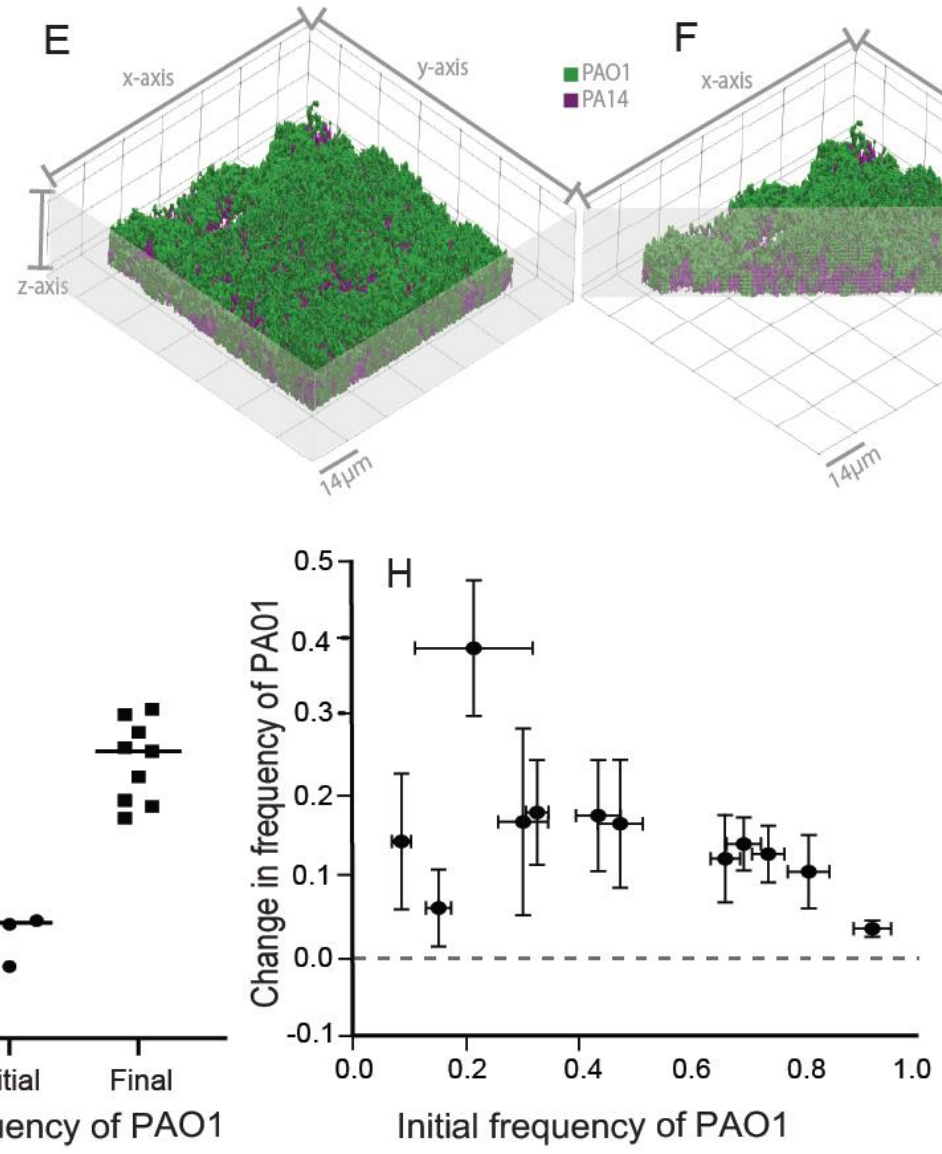

Initial frequency of PAO1

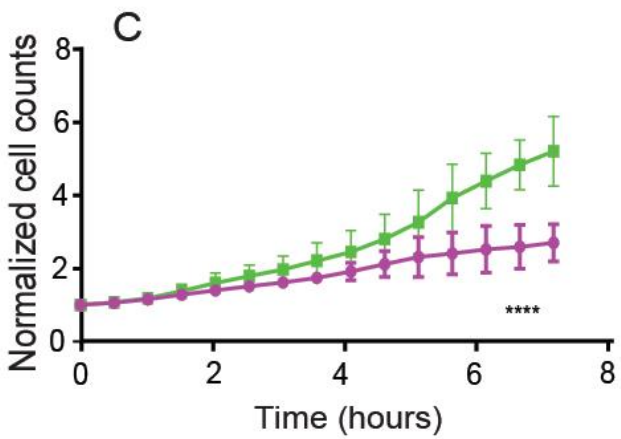

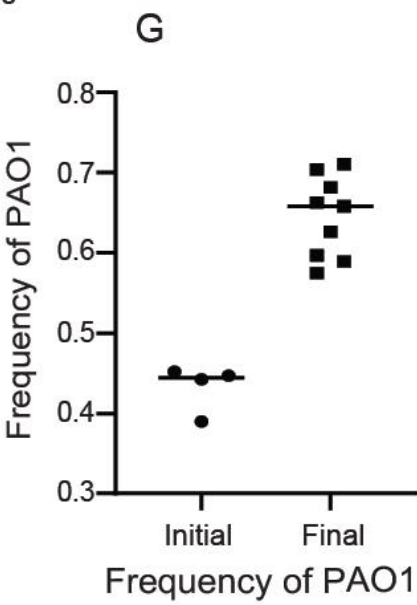

165 Figure 1: PA14-PAO1 dual strain biofilm dynamics. (A,B,D) Representative widefield

166 fluorescence (A,B) and confocal images (D) of PA14-PAO1 dual strain biofilms at the indicated

167 time. The same color scheme for PAO1 (green) and PA14 (magenta) is used in all panels here,

168 and in all figures in the manuscript. (C) Quantification of early biofilm formation (0-7 hrs). $\mathrm{N}=$ 1693 , **** $\mathrm{P}<0.0001$ after $5.5 \mathrm{hr}$. (E) 3-D rendering of the PA14-PAO1 dual strain biofilm. 
170 Rendering is performed as detailed in the Materials and Methods. (F) Cut-away 3-D rendering of

171 the same biofilm in panel (E), showing its internal structure. (G) Initial (0 h) and final (24 h)

172 frequency of PAO1 in a dual strain PA14-PAO1 biofilm grown at $37^{\circ} \mathrm{C}$. (H) Change in

173 frequency of strain PAO1 as a function of its initial frequency in dual strain biofilms of wild-type

174 PAO1 and PA14. Initial frequencies ( $\mathrm{n}=4$ for each data point) and changes in final frequency

175 ( $\mathrm{n}=9$ for each data point) after 24 hours at $37^{\circ} \mathrm{C}$ are plotted. Error bars represent standard

176 deviation.

\section{Psl is required for PAO1 to outcompete PA14 in dual strain biofilms}

We have provided evidence that PAO1 robustly outcompetes PA14 when both are grown together in biofilms. To clarify the mechanism of these dynamics, we attempted to alter the competition outcome (PAO1 dominance) by reducing the ability of PAO1 to compete or genetically manipulating PA14 to enhance its ability to produce biofilms. P. aeruginosa

184 produces three extracellular polysaccharides (EPS) known to facilitate biofilm formation: Pel, 185 Psl and alginate (37). Alginate is broadly conserved in pseudomonads but only conditionally 186 expressed in PAO1 and PA14 during periods of stress (38-40). PAO1 and PA14 both produce 187 Pel, while Psl is unique to PAO1 $(41,42)$. Previous work has also shown that Psl is a cooperative resource among secreting cells, and cells that do not produce it were excluded and outcompeted in a PAO1 background (36). We hypothesized that Psl provided an advantage for PAO1 not afforded to PA14 and tested a PAOl mutant with a clean deletion of the psl promoter (referred to as PAO1 $\Delta p s l$ here) against PA14 in a biofilm. Compared to the WT PAO1 strains, the PAO1

$192 \Delta p s l$ mutant exhibited a significant decrease in relative abundance in competition with PA14 193 after 24h (Fig. 2, Fig. S3A,B).

195 We next used a variety of strategies to enhance PA14 biofilm formation and asked whether these 196 altered PA14 strains could better compete versus PAO1. First, we took advantage of the 197 observation that type 4 pili (T4P) have been shown to mediate initial attachment by $P$. 198 aeruginosa (14), and recent work showed that manipulation of T4P functions could 199 enhance surface commitment by PA14 (32). Specifically, we tested if the PA14 $\Delta$ pilU mutant, a 
strain that is hyper-piliated, shows high constitutive cAMP signaling and rapidly colonizes a

201 surface, would impart a competitive advantage when grown in a biofilm with PAO1. The PA14 $\Delta$ pilU mutant was, similarly to wild-type PA14, outcompeted by PAO1 (Fig. 2).

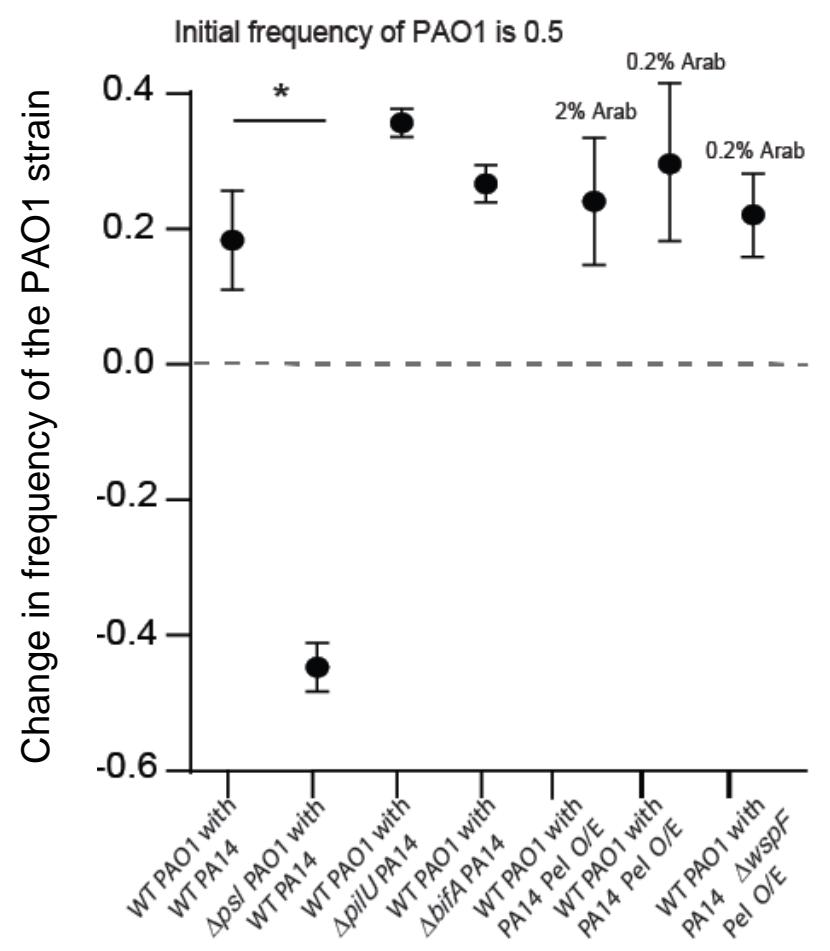

Figure 2: Biofilm mutant analysis. Change in frequency of WT PAO1 and the PAO1 $\Delta p s l$ mutant in the biofilm with WT PA14, as well as change in frequency of WT PAO1 in a dual strain biofilm with PA14 $\Delta$ pilU, PA14 $\Delta$ bifA and Pel over-producing strains. Initial frequency of all strain combinations was 0.5 (n=5-9). Error bars represent standard deviation; Mann-Whitney tests were used for all pairwise comparisons, * denotes $\mathrm{p}=0.0010$, and all other comparisons were not significant.

Next, having shown that Psl plays an important role in the ability for PAO1 to compete against

212 PA14, we reasoned that PA14 might gain a competitive advantage against PAO1 through the 213 induction of PA14's endogenous EPS systems, Pel. To test this hypothesis, we first used a $\Delta b i f A$ 214 mutant, which we showed previously displays increased c-di-GMP signaling and enhanced Pel 215 polysaccharide production (43), but the PA14 $\Delta$ bifA mutant was not any more successful against 216 PAO1 than WT PA14. As an alternative approach we next used a strain that expresses the pel 
217 operon under the control of an arabinose-inducible promoter (designated Pel O/E). Interestingly,

218 while induction of pel expression with $2 \%$ or $0.2 \%$ arabinose increased the biovolume of this

219 strain in a monoculture biofilm (Fig. S3C), the competitive fitness of the Pel O/E strain induced

220 with high and low arabinose was not enhanced when grown with PAO1 (Fig. 2). Finally, we

221 used a variant of the Pel O/E strain which also over produced c-di-GMP due to a mutation in the

$222 w s p F$ gene (designated Pel $\mathrm{O} / \mathrm{E} \Delta w s p F$ ); again, this strain when induced with 0.2 arabinose did

223 not show enhanced fitness compared to the PA14 parent strain (Fig. 2) despite enhanced biomass

224 in monoculture (Fig. S3C). Taken together, these data show that Psl production is a key factor

225 allowing PAO1 to outcompete PA14, and that enhancing PA14 biofilm production via several

226 different strategies is unable to overcome the advantage displayed for PAO1 during biofilm

227 formation.

\section{PAO1 dominates in an environment marked by perturbation.}

230 It is possible that although PA14 is outcompeted by PAO1 within a growing biofilm, PA14 231 might be better suited to dispersal to new locations for future biofilm growth. Therefore, we next 232 asked if PAO1 still dominated in an environment marked by perturbation. To test this idea, we 233 grew a 1:1 mixture of PA14 and PAO1 in microfluidic chambers under flow for a fixed time 234 (either $20 \mathrm{~h}$ or $3 \mathrm{~h}$ ) after which we introduced a disturbance event. For each such event, the 235 outflow tube from the first microfluidic chamber was attached to a second previously 236 uncolonized, clean chamber and the biofilm effluent was used to seed this microfluidic chamber 237 for $2 \mathrm{hrs}$. The goal was to simulate the natural transition of $P$. aeruginosa from an existing
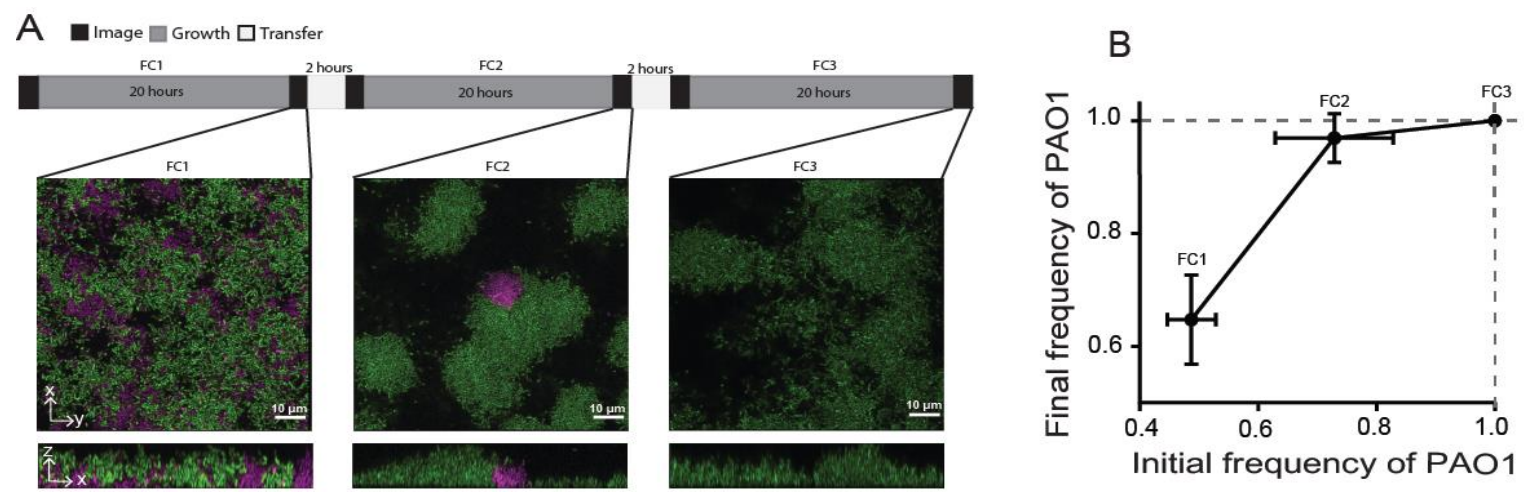

238 biofilm to a new environment with an intervening planktonic phase (Figure 3A, top). 
Figure 3: PAO1 dominates during a perturbation regime. (A) Graphical summary of 240 dispersal experiment regime (top) and representative images (bottom). (B) The frequency

241 dynamics of PAO1 in dispersal experiments through different chambers $(n=3)$. All error bars 242 shown are standard deviation.

243 In dual-strain biofilms that were inoculated 1:1 and grown undisturbed (also shown above), 244 PAO1 accounted for $\sim 75 \%$ of the population at the end of the 20 -h incubation (Fig. 2,3A,3B). In 245 comparison, with disturbance events at $20 \mathrm{~h}$ intervals, the percentage of PAO1 drastically 246 increased compared to PA14 in chamber 2 (after 1 disturbance) to about $97 \%$ of biofilm 247 biovolume. After the second disturbance, PAO1 comprised $\sim 100 \%$ of the biofilm by chamber 3 248 (Fig. 3A, 3B). These data indicate that the disturbance regime still favors PAO1 versus PA14 249 when the two strains compete to form biofilms in successive environmental patches following 250 dispersal.

251 To test if this observed equilibrium in chamber 3 (after two disturbances) was a function of 252 PAO1 having overgrown PA14 (Fig. 1D,1E,1F), we repeated this disturbance assay in 3-h 253 biofilms; at this early time point PAO1 has not yet had sufficient time to overgrow PA14. As 254 there were relatively few cells attaching at $3 \mathrm{hrs}$, quantifying population frequencies was not 255 possible post dispersal as was done for the $20 \mathrm{hr}$ perturbation experiments, so we instead 256 calculated the frequencies observed in chambers two and three after $3 \mathrm{~h}$ of growth (Fig 3B), and 257 then then inoculated new chambers with the same frequency but at a 500-fold higher density. 258 Similar to the trend in 20-h disturbance experiments we saw an increase in PAO1 frequency in 259 the subsequent chamber at $\sim 60 \%$ in chamber 2 and $\sim 70 \%$ in chamber 3 (Fig. S4), again 260 consistent with PAO1 outcompeting PA14 in a fluctuating environment requiring re-colonization 261 of new surfaces.

\section{PA14 is more proficient at invading pre-formed biofilm of PAO1}

264 The data above suggest that PAO1 can dominate PA14 when in competition to colonize a new 265 surface, or under conditions of repeated environmental perturbation. These observations left 266 open the question as to what condition or conditions might PA14 dominate over PAO1, and we 
267 next hypothesized that while PAO1 excels in colonizing new environments and competing in 268 them thereafter, perhaps PA14 has an advantage when colonizing areas in which pre-existing 269 biofilms reside.

270 To test this hypothesis, we grew a biofilm of one of the strains, which we refer to as the "resident 271 strain", in a microfluidic chamber. After $12 \mathrm{~h}$ of growth of the resident strain, we introduced the 272 second strain, referred to as "invader", for 4 hours to assess its ability to colonize and integrate 273 into the resident biofilm.

274 By visual inspection alone it was evident that PAO1 showed minimal invasion into resident 275 PA14 biofilms (Fig. 4A), while PA14 was considerably more proficient at invading pre-formed 276 biofilms of PAO1 (Fig. 4B). To quantify invasion efficiency by PAO1 and PA14, we 277 normalized the fluorescent intensity for each strain at the start of widefield fluorescence imaging 278 (0h) and measured the fold change in signal intensity over $4 \mathrm{~h}$ of the invasion assay. PA14 279 invades resident PAO1 biofilms rapidly after introduction to the chamber (Fig. $\mathbf{4 B}, \mathbf{4 C})$, and by 280 the end of the assay, PA14 invading biovolume was $\sim 3$ orders of magnitude higher than that of
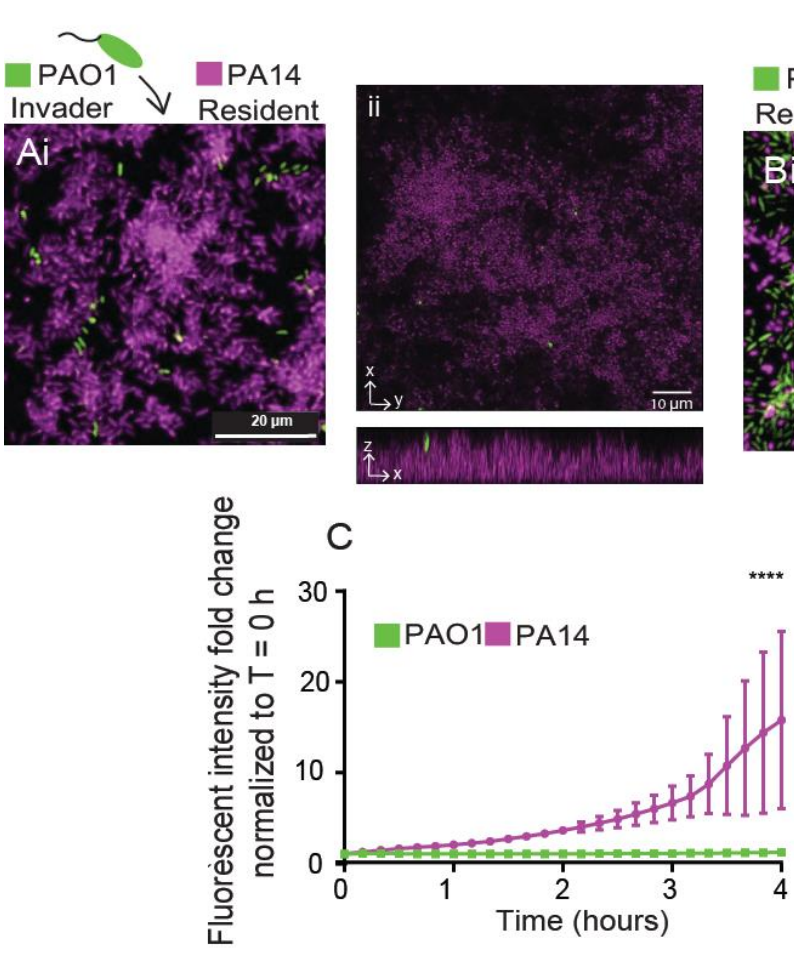
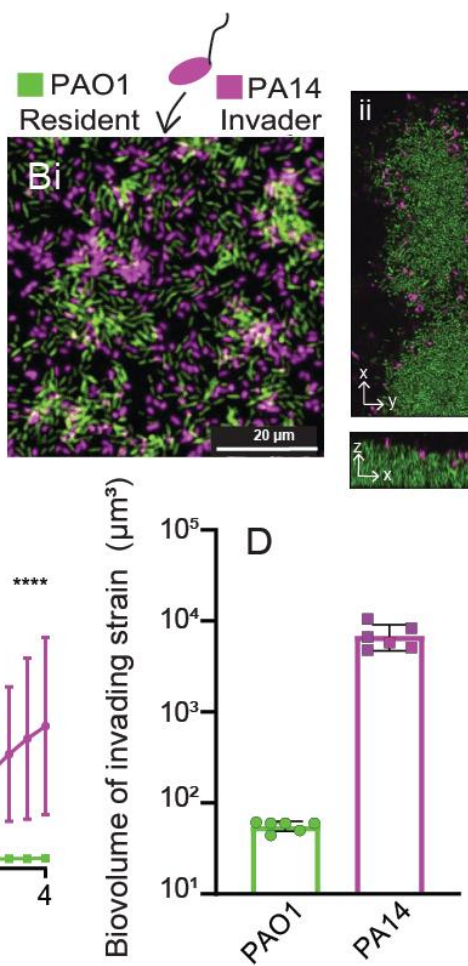

281 PAO1 invading a resident PA14 biofilm, as measured by confocal microscopy (Fig. 4D). 
Figure 4: Invasion dynamics of resident biofilms. (A) Resident biofilms of PA14 were grown for $12 \mathrm{~h}$ and invaded by PAO1 for $4 \mathrm{~h}$ (i) widefield fluorescence image (ii) confocal images with $\mathrm{x}-\mathrm{y}$ optical section above and z projection below. (B) Resident PAO1 biofilms were invaded by 286 PA14. (i) widefield fluorescence image (ii) confocal images with $x-y$ optical section above and z 287 projection below. (C) The invasion efficiency of PA14 (purple) and PAO1 (green) was measured 288 over time by normalizing the change in signal intensity from the start of the assay through $4 \mathrm{~h}$ of 289 invasion. $\mathrm{n}=3$, $* * * * \mathrm{P}<0.0001$ after $3 \mathrm{~h}$ by ANOVA with a Sidak post-test. (D) The total invading 290 strain biovolume of PAO1 and PA14 at the end of the invasion assay $(n=6)$.

\section{PA14 maintains surface coverage relative to PAO1 after starvation.}

293 The studies above assess the ability of PAO1 and PA14 to compete in the context of 294 colonization. Given that the supply of nutrients is transient and irregular in most natural 295 environments, we decided to investigate how PAO1 and PA14 would react to nutrient depletion.

296 To address this question, we grew dual strain biofilms for 12 hour and then changed the influent 297 flow to a carbon-free biofilm medium for $4 \mathrm{~h}$. We observed that strain PA14 had higher 298 persistence with only a $\sim 5 \%$ reduction in surface coverage of cells when carbon-free medium 299 was introduced (Fig. 5A, 5B). By contrast, PAO1 exhibited an $\sim 60 \%$ decrease in surface area 300 coverage over the same time period after influent exchange to carbon-free medium (Fig. 5A, 301 5C), suggesting that PAO1 is more likely to disperse under nutrient-limited conditions.

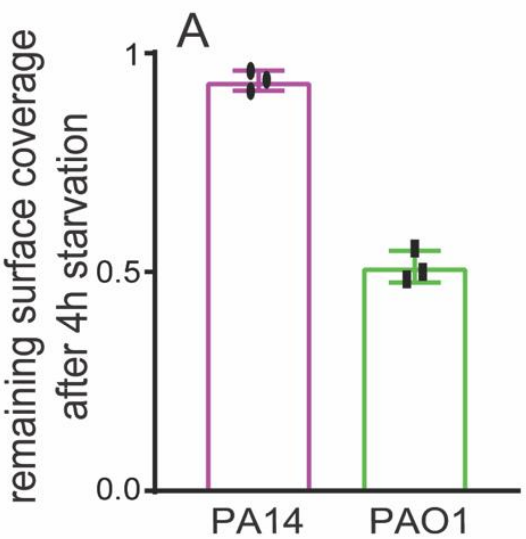

PAO1 PA14

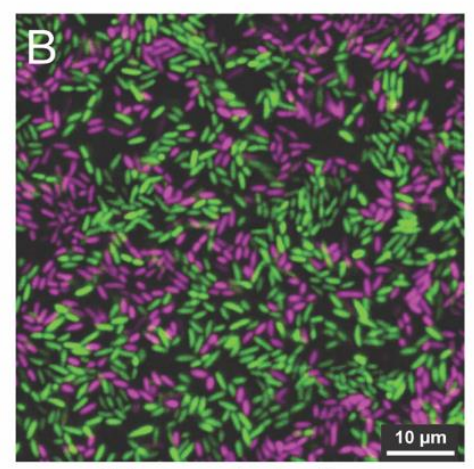

0 hours startvation

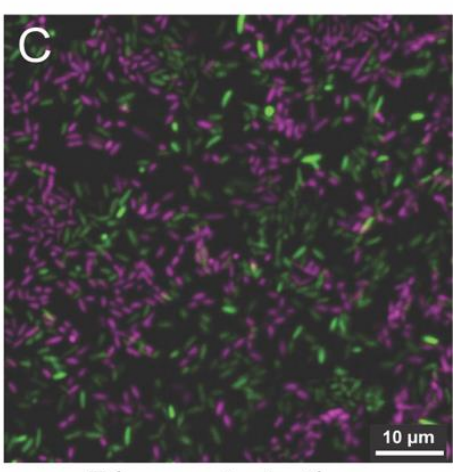

7 hours startvation 
304 Figure 5: Starvation tolerance of PAO1 and PA14 biofilms. Co-culture biofilms of PAO1 and 305 PA14 were grown for $12 \mathrm{~h}$ and deprived of a carbon source (arginine) for $4 \mathrm{~h}$. (A) The surface 306 area occupied by PAO1 and PA14 after 4h starvation was measured as a fraction to the surface 307 area prior to starvation. Error bars represent standard deviation, $n=3$. Representative widefield 308 fluorescence microscopy images of co-culture PAO1 and PA14 biofilms prior to starvation (B) 309 and after 4 h starvation $(\mathbf{C})$.

\section{Discussion}

312 Here we investigated reference strains $P$. aeruginosa PAO1 and PA14 for their ability to 313 compete with each other in the context of biofilm growth. Prior work has provided evidence that 314 PAO1 uses the Wsp system to quickly commit to surfaces through a processive mode of 315 attachment, while PA14 uses the Pil-Chp signaling system to mediate non-processive surface 316 engagement (19). We provide evidence that in microfluidic biofilm culture conditions, PAO1 317 quickly outcompetes and overgrows PA14 in a density and frequency-independent manner. 318 PAO1 and PA14 were also found to spatially segregate on 10 micron length scales, with PA14 319 limited to the substratum and PAO1 over-growing PA14. The competitive advantage of PAO1 is 320 specific to the biofilm mode of growth, as PAO1 did not outgrow PA14 in planktonic mono- or 321 co-culture. Finally, using a regime that mimics environmental perturbation, we show that PAO1 322 remains competitively dominant and indeed reaches fixation in the population after only two 323 recolonization events.

325 To understand how PAO1 outcompetes PA14 we tested a Psl mutant of PAO1 as well as PA14 326 mutants with increased biofilm formation or elevated levels of Pel and found that only through 327 loss of Psl in the PAO1 background did PA14 outcompete PAO1 in biofilm co-culture. 328 Interestingly, while induction of Pel in PA14 led to increased biofilm biomass accumulation in 329 monoculture, increased Pel production did not translate into better competitive ability in mixed 330 culture, a result which suggests that Psl may provide a unique advantage for PAO1 in biofilm 331 environments. The importance of Psl has also been observed in polymicrobial biofilms; 332 consistent with our data, the loss of Psl resulted in a significant decrease in the biovolume of 
333 PAO1 when grown with other species such as Pseudomonas protegens and Klebsiella 334 pneumoniae (44).

We did note two conditions where PA14 seemed to be superior in competition against PAO1 in biofilm co-culture, namely in colonizing a preformed biofilm of PAO1 and in retaining biomass in place in the face of nutrient deprivation. PA14 was better able to invade pre-formed biofilms of PAO1 by $\sim 3$ orders of magnitude than vice versa, suggesting that PA14 may be better suited to exploiting previously colonized environments, while PAO1 is superior in competition for space and resources on unoccupied surfaces. Furthermore, PA14 may be more suited to retaining

342 a grip on space it has occupied when nutrients run low, while PAO1 is more inclined to disperse.

343 The relative of advantage of staying in place under starvation conditions depends on the 344 prevailing environmental conditions: if local nutrient supply fluctuates, then remaining in place 345 may be the better strategy, but if nutrient supply does not return once depleted, then dispersal 346 will be optimal. Recent genomic analysis has found that strains similar to PA14 predominant among CF-derived isolates, while the PAO1-like strains are more likely to be encountered among environmental isolates (45). We speculate on the basis of our results that PA14 has evolved a surface occupation strategy best suited to taking advantage of previously colonized surfaces and commitment to staying in place under fluctuating nutrient conditions, while PAO1 optimizes rapid exploitation of unoccupied surfaces followed by rapid dispersal under nutrient limitation to find new locations for future growth. Thus, as we had predicted in our previous

353 study (19), the differential colonization strategies of PAO1 and PA14 do indeed provide competitive advantages in different contexts.

\section{$356 \quad$ Materials and Methods}

Microfluidic device assembly. The microfluidic devices were made by bonding polydimethylsiloxane (PDMS) chamber molds to size \#1.5 cover glass slips $(60 \mathrm{~mm}$ X $36 \mathrm{~mm}$

360 [LxW], Thermo-Fisher, Waltham MA) using standard soft lithography techniques (46). Each 361 PDMS mold contained 4 chambers, each of which measured $3000 \mu \mathrm{m}$ x $500 \mu \mathrm{m} \times 75 \mu \mathrm{m}$ 362 (LxWxD). To establish flow in these chambers, medium was loaded into $1 \mathrm{~mL}$ BD plastic 363 syringes with 25-gauge needles. These syringes were joined to \#30 Cole-Parmer PTFE tubing 
364 (inner diameter $0.3 \mathrm{~mm}$ ), which was connected to pre-bored holes in the microfluidic device.

365 Tubing was also placed on the opposite end of the chamber to direct the effluent to a waste 366 container. Syringes were mounted to syringe pumps (Pico Plus Elite, Harvard Apparatus), and 367 flow was maintained at $0.1 \mu \mathrm{L}$ per min for all experiments.

Biofilm growth. Overnight cultures of $P$. aeruginosa strains were grown at $37^{\circ} \mathrm{C}$ shaking in lysogeny broth (LB) prior to the start of biofilm experiments. Cultures were normalized to an $\mathrm{OD}_{600}$ of 1 in KA biofilm (47) medium containing 50mM Tris- $\mathrm{HCl}\left(\mathrm{pH} \mathrm{7.4),} 0.6 \mathrm{mM} \mathrm{MgSO}_{4}\right.$, $1.0 \mathrm{~K}_{2} \mathrm{HPO}_{4}$ and $0.4 \%$ arginine. If dual strain biofilms were assessed, equal volumes of cultures adjusted to $\mathrm{OD}_{600}$ of 1 were mixed and used as the inoculum for the microfluidic chamber (completely filling its inner volume), and then the bacteria were allowed to rest for $1 \mathrm{~h}$ at room temperature to permit cells to attach to the glass surface. For the experiments with varied initial frequencies, after cultures of each strain were adjusted to an $\mathrm{OD}_{600}$ of 1 , ratios of the two cultures were added to obtain the desired frequency prior to inoculation. After resting for 1 hour to allow bacterial attachment, the devices were run at $0.5 \mu \mathrm{L}$ per min at $37^{\circ} \mathrm{C}$ and imaged by widefield or confocal microscopy (see below) at time intervals that varied per experiment as noted in the main text. At every sampling time point, images were acquired from nonoverlapping locations within each biofilm chamber. All experiments were repeated with at least 3 biological replicates with 3 or more technical replicates on different days. Total replicates for each experiment are noted in the figure legends for each data set in the text and Supplementary Information.

For the perturbation studies, a dual strain biofilm (1:1 initial frequency of PA14: PAO1) was used to seed a biofilm for either 24 hours or 3 hours, and a $0.5-\mathrm{cm}$ length of tubing was connected to the outlet channel. At every sampling time point, images were acquired from nonoverlapping locations within each biofilm chamber. The outlet chamber was then allowed to seed a new chamber; this process was repeated two times for the 24 hour incubation studies. For the 3-hour protocol, because of low cell numbers attaching and the inability to quantify those low

391 biovolumes, we conducted the disturbance protocol and tabulated the frequency of the two 392 strains in the newly colonized chamber. We then inoculated a new chamber with the same 393 frequency with about 500-fold increased density to allow sufficient biomass to accumulate to determine the relative frequency of each strain. 
396 For invasion experiments, we grew the resident biofilm for $12 \mathrm{~h}$ at a media flow rate of $0.5 \mu \mathrm{L}$

397 per min at $37^{\circ} \mathrm{C}$. After which we introduced the invading strain (adjusted to an $\mathrm{OD}_{600}=1$ ) at the

398 same flow rate for 4 hours. At every sampling time point, images were acquired from

399 nonoverlapping locations within each biofilm chamber.

Microscopy and image analysis. Biofilms in the microfluidic chambers were imaged using a

Zeiss LSM 880 confocal microscope with a 40x/1.2NA or 10x/0.4NA water objective. A 543-nm

laser line was used to excite $\mathrm{mKO}-\kappa$, and a 594-nm laser line was used to excite mKate2. A 458$\mathrm{nm}$ laser line was used to excite Wisteria floribunda lectin stain in the case of Pel quantification experiments. All quantitative analysis of microscopy data was performed using BiofilmQ (48).

Statistics. All statistical analyses were performed in GraphPad Prism. All reported pairwise comparisons were performed using Wilcoxon signed-ranks tests, and multiple comparisons were

411 standard deviation unless otherwise noted.

413 Acknowledgements. This work was supported by funding from the NIH (R37- AI83256-06) to 414 GAO. CDN was supported by the Cystic Fibrosis Foundation (STANTO19RO), NSF (MCB415 1817342) and NIH (P30-DK117469).

\section{Literature Cited.}

419 1. Jahid IK, Ha S Do. 2012. A review of microbial biofilms of produce: Future challenge to 420 food safety. Food Sci Biotechnol 21:299-316.

421 2. Donlan RM. 2001. Biofilms and device-associated infections. Emerg Infect Dis 7:277$422 \quad 281$.

423 3. Van Acker H, Van Dijck P, Coenye T. 2014. Molecular mechanisms of antimicrobial 424 tolerance and resistance in bacterial and fungal biofilms. Trends Microbiol 22:326-333.

425 4. Matz C, McDougald D, Moreno AM, Yung PY, Yildiz FH, Kjelleberg S. 2005. Biofilm 
formation and phenotypic variation enhance predation-driven persistence of Vibrio cholerae. Proc Natl Acad Sci U S A 102:16819-16824.

428 5. Gambino M, Cappitelli F. 2016. Mini-review: Biofilm responses to oxidative stress. $429 \quad$ Biofouling 32:167-178.

430 6. Valentini M, Filloux A. 2016. Biofilms and cyclic di-GMP (c-di-GMP) signaling: Lessons from Pseudomonas aeruginosa and other bacteria. J Biol Chem 291:12547-12555.

7. Fong JCN, Yildiz FH. 2008. Interplay between cyclic AMP-cyclic AMP receptor protein and cyclic di-GMP signaling in vibrio cholerae biofilm formation. J Bacteriol 190:66466659.

8. Passos da Silva D, Schofield MC, Parsek MR, Tseng BS. 2017. An update on the sociomicrobiology of quorum sensing in gram-negative biofilm development. Pathogens 6:1-9.

9. Pellett S, Bigley D V., Grimes DJ. 1983. Distribution of Pseudomonas aeruginosa in a riverine ecosystem. Appl Environ Microbiol 45:328-332.

10. Green SK, Schroth MN, Cho JJ, Kominos SD, Vitanza-Jack VB. 1974. Agricultural plants and soil as a reservoir for Pseudomonas aeruginosa. Appl Microbiol 28:987-991.

11. Romling U, Wingender J, Muller H, Tummler B. 1994. A major Pseudomonas aeruginosa clone common to patients and aquatic habitats. Appl Environ Microbiol 60:1734-1738.

12. Moreau-Marquis S, Stanton BA, O'Toole GA. 2008. Pseudomonas aeruginosa biofilm formation in the cystic fibrosis airway. Pulm Pharmacol Ther 21:595-599.

13. Toutain CM, Caizza NC, Zegans ME, O’Toole GA. 2007. Roles for flagellar stators in biofilm formation by Pseudomonas aeruginosa. Res Microbiol 158:471-477.

14. O’Toole GA, Kolter R. 1998. Flagellar and twitching motility are necessary for Pseudomonas aeruginosa biofilm development. Mol Microbiol 30:295-304.

16. Zhao K, Tseng BS, Beckerman B, Jin F, Gibiansky ML, Harrison JJ, Luijten E, Parsek Pseudomonas aeruginosa biofilms. Nature 497:388-391. 
aeruginosa biofilm formation and architecture. Appl Environ Microbiol 77:5238-5246.

18. Armbruster CR, Parsek MR. 2018. New insight into the early stages of biofilm formation. Proc Natl Acad Sci U S A 115:4317-4319.

19. Lee CK, Vachier J, de Anda J, Zhao K, Baker AE, Bennett RR, Ambruster CR, Lewis KA, Tarnapol RL, Lomba CJ, Hogan DA, Parsek MR, O'Toole GA, Golestanian R, Wong GCL. 2020. Social cooperativity of bacteria during reversible surface attachment in young biofilms: A quantitative comparison of Pseudomonas aeruginosa PA14 and PAO1. MBio $11: 1-21$.

20. Hengge R. 2009. Principles of c-di-GMP signalling in bacteria. Nat Rev Microbiol 7:263273.

21. Jones CJ, Utada A, Davis KR, Thongsomboon W, Zamorano Sanchez D, Banakar V, Cegelski L, Wong GCL, Yildiz FH. 2015. C-di-GMP regulates motile to sessile transition by modulating MshA pili biogenesis and near-surface motility behavior in Vibrio cholerae. PLoS Pathog 11:1-27.

22. Güvener ZT, Harwood CS. 2007. Subcellular location characteristics of the Pseudomonas aeruginosa GGDEF protein, WspR, indicate that it produces cyclic-di-GMP in response to growth on surfaces. Mol Microbiol 66:1459-1473.

23. McDonough KA, Rodriguez A. 2012. The myriad roles of cyclic AMP in microbial pathogens: From signal to sword. Nat Rev Microbiol 10:27-38.

24. Luo Y, Zhao K, Baker AE, Kuchma SL, Coggan KA, Wolfgang MC, Wong GCL, O'Toole GA. 2015. A hierarchical cascade of second messengers regulates Pseudomonas aeruginosa Surface Behaviors. MBio 6:1-11.

25. O’Connor JR, Kuwada NJ, Huangyutitham V, Wiggins PA, Harwood CS. 2012. Surface sensing and lateral subcellular localization of WspA, the receptor in a chemosensory-like system leading to c-di-GMP production. Mol Microbiol 86:720-729.

26. Hickman JW, Tifrea DF, Harwood CS. 2005. A chemosensory system that regulates biofilm formation through modulation of cyclic diguanylate levels. Proc Natl Acad Sci U Søgaard-Andersen L. 2020. PilY1 and minor pilins form a complex priming the type IVa pilus in Myxococcus xanthus. Nat Commun 11:1-14. 
28. Kuchma SL, Ballok AE, Merritt JH, Hammond JH, Lu W, Rabinowitz JD, O’Toole GA. 2010. Cyclic-di-GMP-mediated repression of swarming motility by Pseudomonas aeruginosa: The pilY1 gene and its impact on surface-associated behaviors. J Bacteriol 192:2950-2964.

29. Webster SS, Lee CK, Schmidt WC, Wong GCL. 2021. Fine tuning cyclic-di-GMP signaling in Pseudomonas aeruginosa using the type 4 pili alignment complex. https://www.biorxiv.org/content/10.1101/2020.10.17.343988v1.

30. Freschi L, Vincent AT, Jeukens J, Emond-Rheault JG, Kukavica-Ibrulj I, Dupont MJ, Charette SJ, Boyle B, Levesque RC. 2019. The Pseudomonas aeruginosa pan-genome provides new insights on its population structure, horizontal gene transfer, and pathogenicity. Genome Biol Evol 11:109-120.

32. Lee CK, De Anda J, Baker AE, Bennett RR, Luo Y, Lee EY, Keefe JA, Helali JS, Ma J,

31. Armbruster CR, Lee CK, Parker-Gilham J, de Anda J, Xia A, Tseng BS, Hoffman LR, Jin

33. Choi KH, Schweizer HP. 2006. Mini-Tn7 insertion in bacteria with single att $\operatorname{Tn} 7$ sites:

34. Parsek MR, Tolker-Nielsen T. 2008. Pattern formation in Pseudomonas aeruginosa biofilms. Curr Opin Microbiol 11:560-566.

35. Teal TK, Lies DP, Wold BJ, Newman DK. 2006. Spatiometabolic stratification of Shewanella oneidensis biofilms. Appl Environ Microbiol 72:7324-7330.

36. Irie Y, Roberts AEL, Kragh KN, Gordon VD, Hutchison J, Allen RJ, Melaugh G, Bjarnsholt T, West SA, Diggle SP. 2017. The Pseudomonas aeruginosa PSL polysaccharide is a social but noncheatable trait in biofilms. MBio 8:1-13.

37. Franklin MJ, Nivens DE, Weadge JT, Lynne Howell P. 2011. Biosynthesis of the

518 38. Goldberg JB, Gorman WL, Flynn JL, Ohman DE. 1993. A mutation in algN permits trans 
activation of alginate production by AlgT in Pseudomonas species. J Bacteriol 175:13031308.

39. Wozniak DJ, Wyckoff TJO, Starkey M, Keyser R, Azadi P, O’Toole GA, Parsek MR. 2003. Alginate is not a significant component of the extracellular polysaccharide matrix of PA14 and PAO1 Pseudomonas aeruginosa biofilms. Proc Natl Acad Sci U S A 100:79077912.

40. Sabra W, Kim EJ, Zeng AP. 2002. Physiological responses of Pseudomonas aeruginosa PAO1 to oxidative stress in controlled microaerobic and aerobic cultures. Microbiology 148:3195-3202.

528 41. Friedman L, Kolter R. 2004. Two genetic loci produce distinct carbohydrate-rich structural components of the Pseudomonas aeruginosa biofilm matrix. J Bacteriol 186:4457-4465.

42. Friedman L, Kolter R. 2004. Genes involved in matrix formation in Pseudomonas aeruginosa PA14 biofilms. Mol Microbiol 51:675-690.

43. Kuchma SL, Brothers KM, Merritt JH, Liberati NT, Ausubel FM, O’Toole GA. 2007. BifA, a cyclic-di-GMP phosphodiesterase, inversely regulates biofilm formation and swarming motility by Pseudomonas aeruginosa PA14. J Bacteriol 189:8165-8178.

44. Periasamy S, Nair HAS, Lee KWK, Ong J, Goh JQJ, Kjelleberg S, Rice SA. 2015. Pseudomonas aeruginosa PAO1 exopolysaccharides are important for mixed species biofilm community development and stress tolerance. Front Microbiol 6:1-10.

45. Ozer EA, Nnah E, DIdelot X, Whitaker RJ, Hauser AR, Ochman H. 2019. The population structure of Pseudomonas aeruginosa is characterized by genetic isolation of exoU+ and exoS+ lineages. Genome Biol Evol 11:1780-1796.

46. Ng JMK, Stroock AD, Whitesides GM. 2010. Components for integrated poly

544 47. Collins AJ, Pastora AB, Jarrod Smith T, Dahlstrom K, O’Toole GA. 2020. MapA, a second large RTX adhesin, contributes to biofilm formation by Pseudomonas fluorescens.

547 48. Hartmann R, Jeckel H, Jelli E, Singh PK, Vaidya S, Bayer M, Rode DKH, Vidakovic L, Díaz-Pascual F, Fong JCN, Dragoš A, Lamprecht O, Thöming JG, Netter N, Häussler S, 
bioRxiv preprint doi: https://doi.org/10.1101/2021.05.17.444588; this version posted May 18, 2021. The copyright holder for this preprint (which

was not certified by peer review) is the author/funder, who has granted bioRxiv a license to display the preprint in perpetuity. It is made available under aCC-BY-NC 4.0 International license. 